\title{
Resistance training improves skeletal muscle insulin sensitivity in elderly offspring of overweight and obese mothers
}

\author{
Marco Bucci $^{1}$ - Ville Huovinen ${ }^{1,2,3}$ • Maria Angela Guzzardi ${ }^{4}$ Suvi Koskinen ${ }^{1}$. \\ Juho R. Raiko ${ }^{1}$ - Heta Lipponen ${ }^{1}$ - Shaila Ahsan ${ }^{1}$ - Robert M. Badeau ${ }^{1}$. \\ Miikka-Juhani Honka ${ }^{1}$ • Jukka Koffert ${ }^{1}$ - Nina Savisto ${ }^{1}$ • Minna K. Salonen ${ }^{5,6}$. \\ Jonathan Andersson $^{7}$ - Joel Kullberg ${ }^{7}$. Samuel Sandboge ${ }^{5,6}$ • Patricia Iozzo ${ }^{1,4}$. \\ Johan G. Eriksson ${ }^{5,6,8,9} \cdot$ Pirjo Nuutila ${ }^{1,10}$
}

Received: 4 June 2015 / Accepted: 14 September 2015 / Published online: 21 October 2015

(C) Springer-Verlag Berlin Heidelberg 2015

\begin{abstract}
Aims/hypothesis Maternal obesity predisposes offspring to adulthood morbidities, including type 2 diabetes. Type 2 diabetes and insulin resistance have been associated with shortened telomere length. First, we aimed to investigate whether or not maternal obesity influences insulin sensitivity and its relationship with leucocyte telomere length (LTL) in elderly women. Second, we tested whether or not resistance exercise training improves insulin sensitivity in elderly frail women.

Methods Forty-six elderly women, of whom 20 were frail offspring of lean/normal weight mothers (OLM, BMI $\leq 26.3 \mathrm{~kg} / \mathrm{m}^{2}$ ) and 17 were frail offspring of overweight/ obese mothers (OOM, BMI $\left.\geq 28.1 \mathrm{~kg} / \mathrm{m}^{2}\right)$, were studied before and after a 4 month resistance training (RT) intervention. Muscle insulin sensitivity of glucose uptake was measured using ${ }^{18} \mathrm{~F}$-fluoro-2-deoxyglucose and positron emission tomography with computed tomography during a hyperinsulinaemic-euglycaemic clamp. Muscle mass and lip-
\end{abstract}

Electronic supplementary material The online version of this article (doi:10.1007/s00125-015-3780-8) contains peer-reviewed but unedited supplementary material, which is available to authorised users.

Marco Bucci

marbuc@utu.fi

1 Turku PET Centre, University of Turku, PO BOX 52, FIN-20521 Turku, Finland

2 Department of Radiology, Faculty of Medicine, University of Turku, Turku, Finland

3 Department of Radiology, Medical Imaging Centre of Southwest Finland, Turku University Hospital, Turku, Finland

4 Institute of Clinical Physiology, National Research Council, Pisa, Italy id content were measured using magnetic resonance and LTL was measured using real-time PCR.

Results The OOM group had lower thigh muscle insulin sensitivity compared with the OLM group $(p=0.048)$ but similar whole body insulin sensitivity. RT improved whole body and skeletal muscle insulin sensitivity in the OOM group only ( $p=0.004$ and $p=0.013$, respectively), and increased muscle mass in both groups $(p<0.01)$. In addition, in the OOM group, LTL correlated with different thigh muscle groups insulin sensitivity $(\rho \geq 0.53 ; p \leq 0.05)$. Individuals with shorter LTL showed a higher increase in skeletal muscle insulin sensitivity after training $(\rho \geq-0.61 ; p \leq 0.05)$.

Conclusions/interpretation Maternal obesity and having telomere shortening were associated with insulin resistance in adult offspring. A resistance exercise training programme may reverse this disadvantage among offspring of obese mothers.

Trial registration: ClinicalTrials.gov NCT01931540

5 Folkhälsan Research Centre, Helsinki, Finland

6 Department of Chronic Disease Prevention, National Institute for Health and Welfare, Helsinki, Finland

7 Department of Radiology, Uppsala University, Uppsala, Sweden

8 Department of General Practice and Primary Health Care, University of Helsinki and Helsinki University Hospital, Helsinki, Finland

9 Vaasa Central Hospital, Vaasa, Finland

10 Department of Endocrinology, Turku University Hospital, Turku, Finland 
Keywords Computed tomography $\cdot$ Elderly . Hyperinsulinaemic-euglycaemic clamp · Insulin sensitivity . Magnetic resonance $\cdot$ Maternal obesity $\cdot$ Positron emission tomography $\cdot$ Resistance training $\cdot$ Telomere shortening

\begin{tabular}{|c|c|}
\hline \multicolumn{2}{|c|}{ Abbreviations } \\
\hline $\mathrm{CT}$ & Computed tomography \\
\hline CTR & Non-frail control group \\
\hline DORIAN & $\begin{array}{l}\text { Developmental ORIgins of healthy } \\
\text { and unhealthy AgeiNg }\end{array}$ \\
\hline${ }^{18} \mathrm{~F}-\mathrm{FDG}$ & ${ }^{18} \mathrm{~F}$-fluoro-2-deoxyglucose \\
\hline $\mathrm{fP}$ & Fasting plasma \\
\hline GU & Glucose uptake rate \\
\hline HBCS & Helsinki Birth Cohort Study \\
\hline IMCL & Intramyocellular lipid content \\
\hline $\mathrm{Ki}$ & Net influx rate constant \\
\hline LTL & Leucocyte telomere length \\
\hline MRS & Magnetic resonance spectroscopy \\
\hline OLM & Offspring of lean/normal weight mothers \\
\hline OOM & Offspring of obese/overweight mothers \\
\hline$p^{\wedge}$ & Nonparametric $p$ value \\
\hline PET & Positron emission tomography \\
\hline RT & Resistance training \\
\hline SUV & Standardised uptake value \\
\hline
\end{tabular}

\section{Introduction}

Maternal obesity during pregnancy is associated with an unfavourable developmental environment for the growing fetus [1] and predisposes it to morbidities in later life (fetal programming), including type 2 diabetes, especially in female offspring $[2,3]$. This rules against genetic determinants of disease risk because the in utero nutritional status affects male and female fetuses in different ways [4]. The genetic background of the offspring interacts with intrauterine programming. However, it has been argued that maternal obesity is more important than genetic factors in determining health in adult life both in humans [5, 6] and animal models [7], which have low genetic variability. Furthermore, there is a greater association between maternal BMI and the manifestations of overweight in children compared with paternal BMI [8].

Fetal programming, which can lead to system immaturity and a limited capacity for compensation and repair, has similarities with system exhaustion that is characteristic of ageing. Insulin resistance is associated with ageing and frailty [9]. Frailty is a syndrome characterised by unintentional weight loss, exhaustion, muscle weakness, slow walking speed and low physical activity [10]. The handgrip test is a valid marker for muscle weakness to identify frail individuals in an elderly population $[9,11]$.
Frailty is highly prevalent during old age and confers a high risk for adverse outcomes [10]. Ageing is associated with an impaired insulin action in skeletal muscle [12]. Frailty in elderly women is thus a good model for studying unhealthy ageing and the effect of maternal obesity. Little is known about the relationships between maternal obesity, frailty and insulin resistance.

Telomeres consist of DNA repeat sequences and associated proteins that are present at the end of chromosomes. Leucocyte telomere length (LTL) is a biomarker for biological ageing and is useful for predicting morbidity and mortality [13]. Recently, type 2 diabetes has been associated with shortening of LTL [14]. In addition, risk factors for type 2 diabetes, such as insulin resistance, are associated with shortening of LTL, especially in men [15] and premenopausal women but not in postmenopausal women [16]. This lack of association in postmenopausal women is the focus of this study.

The hyperinsulinaemic-euglycaemic clamp is the gold standard technique to assess insulin sensitivity [17]. In the present study, the glucose clamp technique was used to measure both whole body insulin sensitivity and, in combination with ${ }^{18} \mathrm{~F}$-fluoro-2-deoxyglucose $\left({ }^{18} \mathrm{~F}\right.$-FDG) and positron emission tomography (PET) and computed tomography (CT), thigh muscle glucose uptake during insulin stimulation. MRI and magnetic resonance spectroscopy (MRS) were used to quantify skeletal muscle mass of different muscle groups and intramyocellular lipid content (IMLC).

Although a few studies have examined the effect of resistance training (RT) on whole body insulin sensitivity in elderly humans $[18,19]$, no study has yet investigated the effect of RT on skeletal muscle insulin sensitivity using ${ }^{18} \mathrm{~F}-\mathrm{FDG}$ PET/CT and clamp design in elderly women or the association with LTL.

The current study assessed the role of maternal obesity in glucose metabolism under an insulin-stimulated state and the effects of a RT exercise intervention. The women participating in the RT intervention where in the lowest half of handgrip score of a large birth cohort [2]. This study was part of an EU-funded programme, the Developmental ORIgins of healthy and unhealthy AgeiNg (DORIAN), which aims to investigate the long-term impact of maternal obesity on health of the offspring [20]. We hypothesised that frail elderly women are insulin resistant compared with non-frail women and that frail women, who are the offspring of obese/overweight mothers (OOM), are more insulin resistant than offspring of lean/normal weight mothers (OLM). We also postulated that these frail women, who are insulin resistant, are more sensitive to metabolic improvements achieved with exercise.

\section{Methods}

Participants All study participants were selected from the Helsinki Birth Cohort Study (HBCS) [2], the largest, best- 
characterised longitudinal birth cohort in the world. HBCS includes 13,345 individuals born during 1934-1944. The participants were selected from a sub-cohort of 2,000 individuals, who had been thoroughly clinically characterised throughout the years. Based on the last study visit, 37 elderly women were selected as frail participants and nine elderly women as the non-frail control group (CTR). The criterion for frailty was the handgrip test using the median strength of the whole population (lower half: frail, higher half: non-frail). Data for grip strength measurements were obtained from previously conducted clinical examinations [21].

To investigate the effect of maternal obesity, the frail participants were divided into two groups according to maternal BMI prior to delivery: OOM with BMI $\geq 28.1 \mathrm{~kg} / \mathrm{m}^{2}$ (highest quartile of the entire population) and OLM with BMI $\leq 26.3 \mathrm{~kg} / \mathrm{m}^{2}$ (lower half of the entire population). CTR were also OLM. Individuals with diabetes requiring insulin treatment or fasting plasma (fP) glucose $>7 \mathrm{mmol} / \mathrm{l}$ at the last visit before enrolment were excluded. Individuals currently smoking and those with comorbidities influencing insulin sensitivity and contraindications for participating in an exercise intervention (i.e. chronic atrial fibrillation and pacemaker) or an MRI study were also excluded. The nature and risks of the study were explained and all participants gave their written informed consent. The study protocol was approved by the Ethics Committee of the Hospital District of Southwest Finland and conducted according to the principles of the Declaration of Helsinki.

Study design The study design is depicted in Fig. 1. A frail group of elderly women and a CTR group were studied at baseline, and only the frail group was studied after 4 months of a supervised resistance exercise intervention programme. The intervention consisted of training sessions three times per week for $60 \mathrm{~min}$. Details about the exercise programme are reported in the electronic supplementary material (ESM) Methods. The examinations (both at baseline and after intervention) were performed on two consecutive days. On the first day, MRI and MRS of the legs were performed; on the second day, PET/CT was targeted to the legs using ${ }^{18}$ F-FDG and a hyperinsulinaemic-euglycaemic clamp. Five studies were cancelled following treatment owing to withdrawals of consent (one PET, two magnetic resonance) and technical difficulties (two PET).

MRI and ${ }^{1}$ H-MRS studies During the first day of examinations, the participants underwent a whole body MRI T1weighted scan and an MRS scan of the left calf. Thigh muscle volumes were segmented from the whole body MRI images (ESM Fig. 1) with an automatic procedure (few manual corrections were applied afterwards) as explained in the ESM.

The IMCL was measured with ${ }^{1} \mathrm{H}$-MRS from the tibialis anterior muscle. The MRI/ ${ }^{1} \mathrm{H}-\mathrm{MRS}$ studies were performed
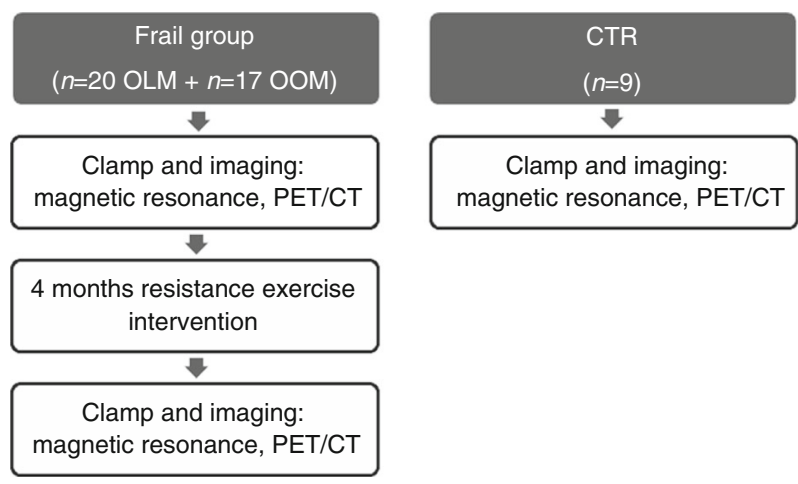

Clamp and imaging: magnetic resonance, PET/CT

Fig. 1 Study design

before and after RT using a 1.5 T MR imager (Gyroscan Intera CV Nova Dual, Philips Medical Systems, Best, the Netherlands) with a flexible surface coil (for the tibialis MRS) and an internal body coil (for the whole-body MRI). The ${ }^{1} \mathrm{H}$-MRS spectra were analysed using the LCModel software package (version 6.3-0C, Linux platform) [22]. Further information can be obtained from our previous publication [23].

PET/CT study The PET/CT study was performed after an overnight fast (12 h). Body fat percentage was measured using a bioelectrical impedance scale (Omron, model HBF-400-E, Omron Healthcare, Lake Forest, IL, USA). Two catheters were inserted: one into the antecubital vein of the right arm for saline infusion and blood sampling, another into the left arm for glucose, insulin and ${ }^{18}$ F-FDG injection. After catheterisation, baseline blood samples were collected. The hyperinsulinaemic-euglycaemic clamp technique was performed as previously described [17]. The rate of insulin infusion was $1 \mathrm{mU} \mathrm{kg}^{-1} \mathrm{~min}^{-1}$ (Actrapid, Novo Nordisk, Copenhagen, Denmark). During hyperinsulinaemia, normoglycaemia was maintained by a variable infusion rate of $20 \%$ glucose based on plasma glucose determinations taken every 5-10 min from arterialised blood. After 40-50 min, when a steady state condition was reached, the participant was moved into the PET/CT scanner (Discovery 690, General Electric (GE) Medical systems, Milwaukee, WI, USA). ${ }^{18} \mathrm{~F}$-FDG was produced with a FASTlab synthesiser (GE Healthcare, Waukesha, WI, USA) in accordance with a modified method described by Hamacher et al [24]. ${ }^{18}$ F-FDG was then administered and the scanning started immediately after tracer injection. The chest area was scanned first for $35 \mathrm{~min}$ (frames $8 \times 15 \mathrm{~s}, 3 \times 60 \mathrm{~s}, 6 \times 300 \mathrm{~s}$ ) to image the heart cavity, and the femoral area was scanned approximately $60 \mathrm{~min}$ after injection for $15 \mathrm{~min}$ (frames $5 \times 180 \mathrm{~s}$ ).

CT scans were obtained in between the PET scans. The arm for blood sampling was warmed up with a heating pillow to arterialise venous blood. During the scan, arterialised venous blood samples were drawn for the determination of blood radioactivity, measured using an automatic gamma counter (Wizard 1480 3, Wallac, Turku, Finland). Insulin and glucose 
concentrations were measured at 0,30,60 and 90 min after the

${ }^{18} \mathrm{~F}$-FDG injection.

PET/CT data processing The software Carimas (v.2.71, Turku PET Centre, Turku, Finland) was used to analyse PET/CT images of thighs. Image data were corrected for dead time, decay and photon attenuation (based on CT images). Regions of interest were drawn manually on the images of each skeletal muscle compartment (quadriceps, adductor magnus, hamstring and adductor longus) of both thighs (Fig. 2a). An anatomical reference on the $\mathrm{CT}$ images was adopted to draw on slices in the same positions.

After obtaining the time activity curves (skeletal muscle and input function), kinetic modelling using the GjeddePatlak graphical method $[25,26]$ was performed to obtain the net influx rate constant (Ki) of ${ }^{18} \mathrm{~F}$-FDG in skeletal muscle. Skeletal muscle glucose uptake (GU) per $\mathrm{kg}$ of tissue was calculated by multiplying Ki by the plasma glucose concentration and dividing by a lumped constant of 1.2 [27]. GU per $\operatorname{depot}(\operatorname{depot} \mathrm{GU}$ ) was calculated as GU (per kg) multiplied for the skeletal muscle group mass. See ESM Methods for further details.

LTL assessment Peripheral blood mononuclear cells were extracted by a whole blood sample with the Ficoll-Hypaque gradient technique [28]. DNA was extracted from peripheral
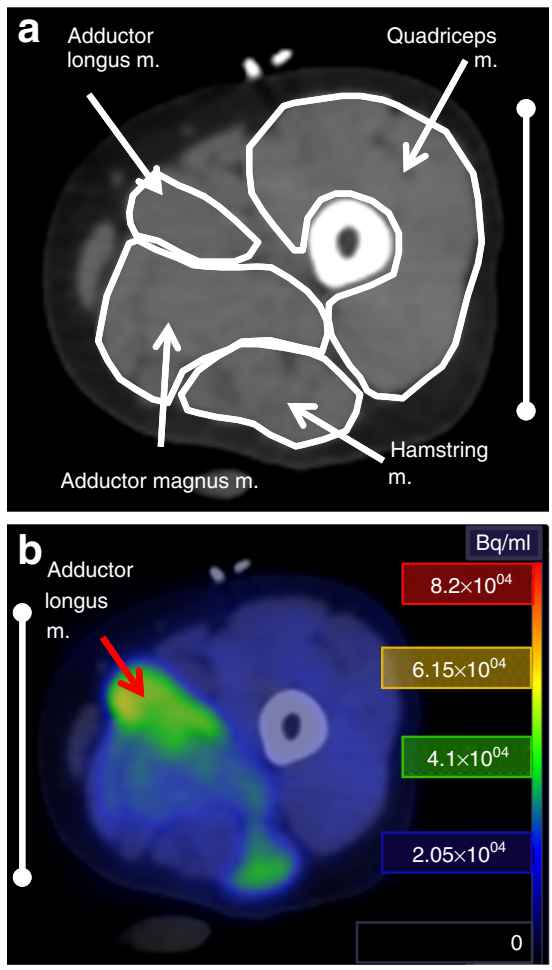

Fig. 2 Thigh cross-sectional (a) CT image with different muscle (m.) compartments and (b) PET image with particularly high FDG activity in the adductor longus muscle. Scale bars, $10 \mathrm{~cm}$ blood mononuclear cells using a commercially available kit according to manufacturer's instruction (DNeasy blood and tissue kit, Qiagen, Milan, Italy). For LTL measurement, DNA concentration was standardised to $4 \mathrm{ng} / \mu \mathrm{l}$ in all samples, and a multiplex quantitative PCR method was used as previously described by Cawthon [29]. The method of assessment of telomere length from peripheral blood mononuclear cells is explained in more detail in the ESM Methods.

Biochemical analysis Plasma glucose during clamp was determined with the glucose oxidase method (Analox GM9 Analyzer, Analox Instruments, London, UK). Serum insulin was measured using an automatised electro-chemiluminescence immunoassay (Cobas 8000, Roche Diagnostics, Mannheim, Germany). HDL-cholesterol, triacylglycerols and fasting glucose were measured with an automatised enzymatic assay (Cobas 8000).

Statistical analysis Statistical analysis was performed using the SPSS IBM 20.0 statistical programme (SPSS, Chicago, IL, USA). Data are reported as mean \pm SE. The Shapiro-Wilk test was used to assess the normality of variables. Student's paired $t$ test was used to compare results before and after training for normally distributed variables and a Wilcoxon test was used for non-normally distributed data. Group comparisons among CTR and frail OOM and OLM were performed using parametric and nonparametric ANOVA tests. For parametric analysis, a Fisher's least significant difference post hoc test was performed. Correction for multiple comparisons was tested with the false discovery rate implemented, as previously described [30]. Correlations were calculated using Spearman's correlation coefficient $(\rho)$. Statistical tests were considered significant with $p \leq 0.05$ (parametric test $p$ value) or $p^{\wedge} \leq 0.05$ (nonparametric test $p$ value). Trends $(p \leq 0.06)$ are reported.

\section{Results}

Cross-sectional results: the role of maternal obesity The frail and non-frail groups were matched for age, BMI, body fat percentage and BP (Table 1). The OOM and OLM groups did not differ.

Whole body insulin sensitivity was not different between OOM, OLM and CTR groups (Fig. 3a), but skeletal muscle insulin sensitivity was significantly lower in the OOM group compared with OLM (Fig. 3b). When the comparison was corrected for BMI, however, the significance was lost $(p=0.077)$.

Analysis of GU separately in different muscle compartments within each group showed that GU (per $\mathrm{kg}$ ) in the quadriceps muscle was the lowest ( $p$ and $p^{\wedge} \leq 0.01$ ), whereas the relatively small adductor longus muscle had high GU (per kg), especially in the OOM group (Fig. 2b, Fig. 4a; 
Table 1 General characteristics at baseline, comparisons between groups

\begin{tabular}{|c|c|c|c|c|c|c|}
\hline & Control group $(n=9)$ & Frail group $(n=37)$ & $p$ & OLM $(n=20)$ & $\operatorname{OOM}(n=17)$ & $p$ \\
\hline Age (years) & $71.4 \pm 0.9$ & $71.9 \pm 0.5$ & 0.97 & $72.3 \pm 0.6$ & $71.5 \pm 0.9$ & 0.26 \\
\hline BMI $\left(\mathrm{kg} / \mathrm{m}^{2}\right)$ & $27.8 \pm 1.4$ & $27.2 \pm 0.8$ & 0.70 & $26.6 \pm 1.1$ & $27.9 \pm 1.1$ & 0.40 \\
\hline WHR & $0.90 \pm 0.01$ & $0.91 \pm 0.01$ & 0.99 & $0.91 \pm 0.02$ & $0.9 \pm 0.01$ & 0.48 \\
\hline Body fat $(\%)$ & $40.1 \pm 1.6$ & $39.5 \pm 1.0$ & 0.77 & $38.6 \pm 1.5$ & $40.5 \pm 1.2$ & 0.35 \\
\hline Systolic BP (mmHg) & $156 \pm 4$ & $162 \pm 3$ & 0.33 & $161 \pm 3$ & $163 \pm 5$ & 0.70 \\
\hline Diastolic BP (mmHg) & $87 \pm 4$ & $90 \pm 2$ & 0.32 & $88 \pm 2$ & $93 \pm 2$ & 0.19 \\
\hline fP-triacylglycerol (mmol/l) & $0.9 \pm 0.1$ & $0.8 \pm 0.0$ & 0.14 & $0.8 \pm 0.1$ & $0.8 \pm 0.1$ & 0.48 \\
\hline fP-HDL-cholesterol (mmol/l) & $1.7 \pm 0.1$ & $1.9 \pm 0.1$ & 0.22 & $1.9 \pm 0.1$ & $1.9 \pm 0.1$ & 0.96 \\
\hline fP-glucose $(\mathrm{mmol} / \mathrm{l})$ & $6.4 \pm 0.3$ & $6.0 \pm 0.1$ & 0.16 & $6.0 \pm 0.2$ & $5.9 \pm 0.2$ & 0.98 \\
\hline fP-insulin (pmol/I) & $64.2 \pm 13.2$ & $57.0 \pm 5.4$ & 0.60 & $57.6 \pm 8.4$ & $56.4 \pm 7.2$ & 0.77 \\
\hline
\end{tabular}

$p^{\wedge} \leq 0.05$ ). In the OLM and CTR group, GU (per kg) was significantly higher in the adductor longus muscle than in the quadriceps and hamstring ( $p \leq 0.05$ for both, in both groups) but not in the adductor magnus muscle (Fig. 4a). Multiple comparisons correction did not change the results.

In the frail study groups, we found that adductor magnus and hamstring muscles $\mathrm{GU}$ were reduced in the OOM group compared with the OLM group (Fig. 4a).

Quadriceps muscles were the biggest muscle group in the portion of thigh muscle examined (Fig. 2a, Fig. 4b), and adductor longus of the OOM group was significantly smaller compared with $\operatorname{OLM}\left(p^{\wedge}=0.033\right)$.
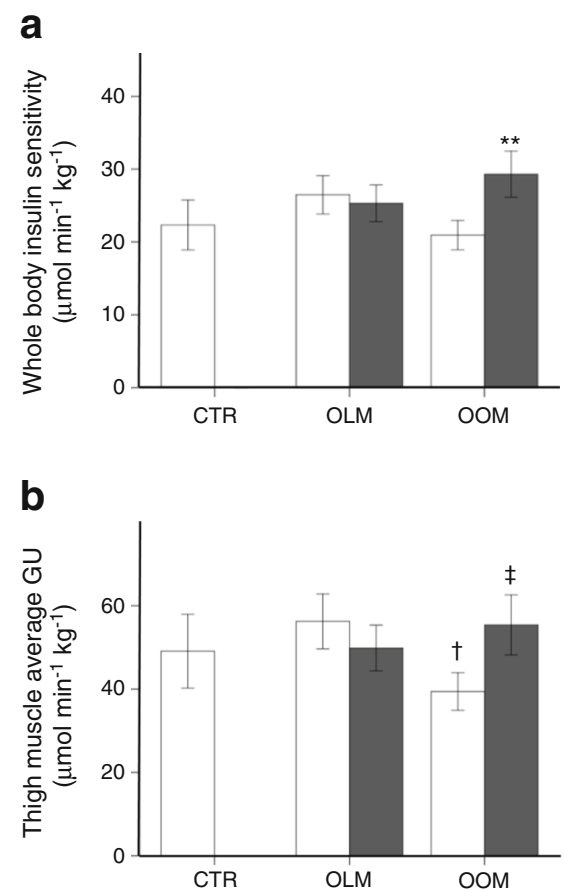

Fig. 3 (a) Whole body and (b) skeletal muscle insulin sensitivity (insulin-stimulated GU) improve in OOM after exercise intervention. GU/kg in (b) is an average of quadriceps, hamstring and adductor magnus muscles. White bars, baseline; black bars, treatment. ${ }^{*} p \leq 0.01$ vs baseline; ${ }^{\ddagger} p^{\wedge} \leq 0.05$ vs baseline; ${ }^{\dagger} p \leq 0.05$ vs OLM baseline
Glucose uptake per kilogram was then multiplied by corresponding muscle masses (segmented by the five-six slices on the CT images) to obtain the glucose uptake depot results (Fig. 4c). The quadriceps muscle group GU (depot) was higher than that of the hamstring and adductor longus muscles but similar to adductor magnus muscle in all three groups (Fig. 4c). GU (depot) of hamstring and adductor magnus muscles was significantly lower in OOM than in OLM $(p=0.018$ and $p^{\wedge}=0.049$, respectively).

Effect of RT on insulin sensitivity The intervention did not change BMI, body fat percentage, BP, fasting circulating lipids, insulin or glucose in either OOM or OLM (Table 2). WHR was reduced after training in the OLM group only $(p=0.05$; Table 2). Exercise increased GU (per kg) of all different compartments except adductor longus muscle only in OOM (Fig. 5c-f). The IMCL, measured with MRS, was decreased after RT exercise in the OOM group only $\left(p^{\wedge}=0.043\right.$; Fig. 6).

RT increased the mass of quadriceps muscles and the adductor longus muscle, and tended to increase the mass of adductor magnus in OOM, and increased quadriceps muscles and tended to increase adductor magnus in OLM (Table 2).

The increase in weight that was obtained with the leg curl exercise (end of treatment vs baseline) correlated positively with the change of hamstring muscles GU (depot; $\rho=0.42$, $\left.p^{\wedge}=0.032, n=26\right)$ and the change of adductor magnus muscle $\mathrm{GU}$ (per kg; $\rho=0.45, p^{\wedge}=0.019, n=27$ ) when grouping all frail individuals.

LTL LTL at baseline was positively associated with GU (per $\mathrm{kg}$ ) of quadriceps, adductor magnus and hamstring muscles $\left(\rho=0.53, p^{\wedge}=0.041 ; \rho=0.56, p^{\wedge}=0.028 ; \rho=0.58, p^{\wedge}=0.023\right.$, respectively) and also with $\mathrm{GU}$ (depot) of adductor magnus and hamstring muscles $\left(\rho=0.66, p^{\wedge}=0.010 ; \rho=0.55\right.$, $p^{\wedge}=0.043$, respectively) in OOM group but not in the other groups. 
Fig. 4 Baseline values of skeletal muscle (a) GU ( $/ \mathrm{kg}$ ), (b) mass and (c) GU (depot), which takes into account mass and GU/kg. In (b) and (c) skeletal muscle refers to a depot of 5-6 slices. White bars, CTR; grey bars, OLM; black bars, OOM. Within group vs quadriceps muscle: ${ }^{*} p \leq 0.05$, $* * * p \leq 0.001 ;{ }^{\dagger \dagger} p^{\wedge} \leq 0.01$, ${ }^{\dagger \dagger} p^{\wedge} \leq 0.001$. Between groups (vs OLM): ${ }^{\star} p \leq 0.05 ;{ }^{\circledR} p^{\wedge} \leq 0.05$. When correcting for BMI, the comparisons between groups remained significant except for GU (depot) in adductor magnus muscle (c). Between group (vs frail group [OLM+OOM] ${ }^{q} p \leq$ 0.05

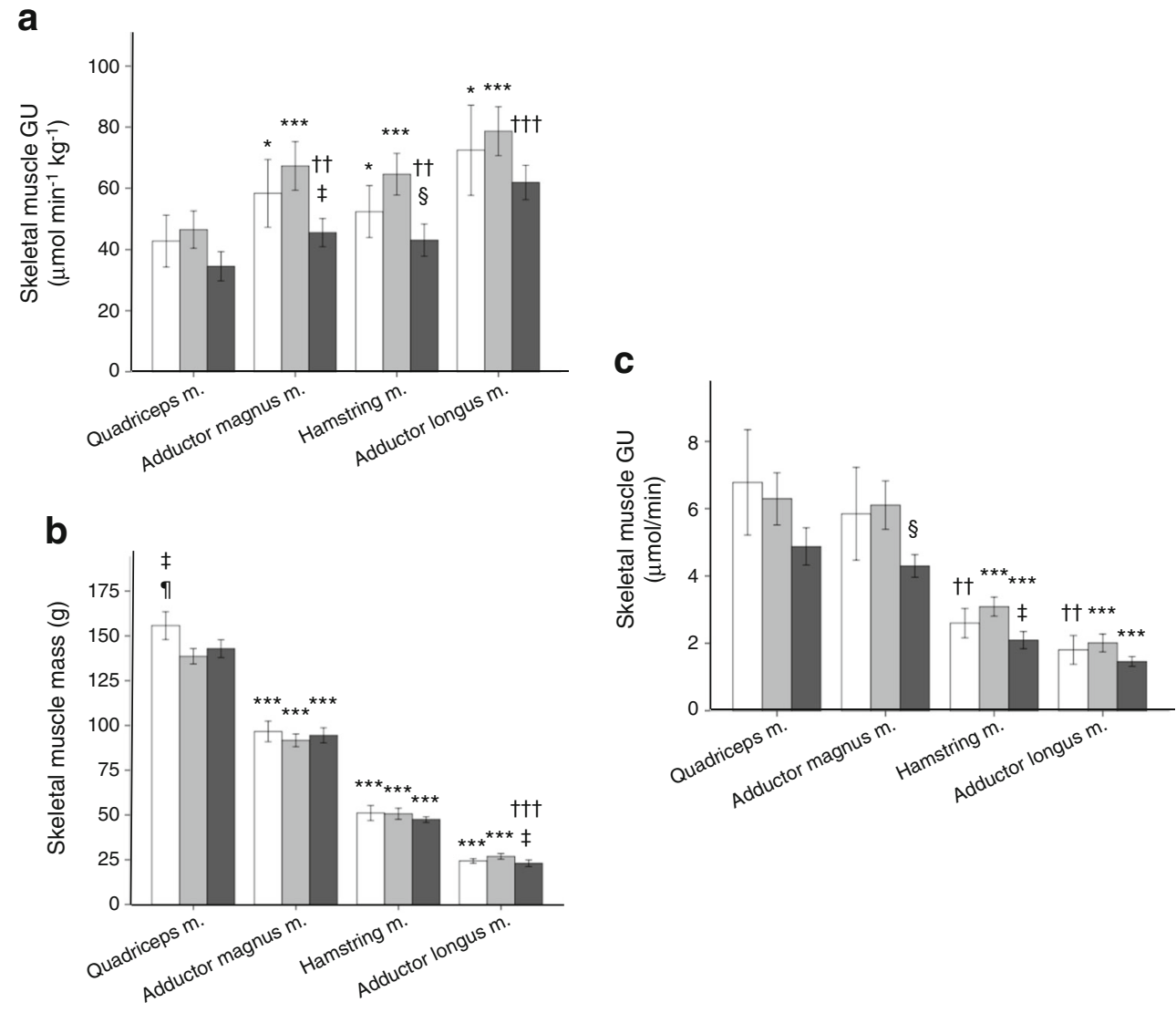

LTL did not change after treatment in either of the two frail groups (OLM and OOM). In the OOM group only, LTL correlated negatively with the change in GU (per kg) of quadriceps, adductor magnus and hamstring muscles $\left(\rho=-0.65, p^{\wedge}=\right.$

Table 2 Effect of exercise on general characteristics and thigh muscle mass in the frail groups sub-divided according to maternal BMI

\begin{tabular}{|c|c|c|c|c|c|c|}
\hline & \multicolumn{2}{|l|}{ OLM } & \multirow[t]{2}{*}{$p$} & \multicolumn{2}{|l|}{ OOM } & \multirow[t]{2}{*}{$p$} \\
\hline & Baseline $(n=20)$ & Exercise $(n=19)$ & & Baseline $(n=17)$ & Exercise $(n=16)$ & \\
\hline BMI $\left(\mathrm{kg} / \mathrm{m}^{2}\right)$ & $26.6 \pm 1.1$ & $27.1 \pm 1.1$ & 0.52 & $27.9 \pm 1.1$ & $27.6 \pm 1.2$ & 0.92 \\
\hline WHR & $0.91 \pm 0.02$ & $0.89 \pm 0.01$ & 0.05 & $0.90 \pm 0.01$ & $0.88 \pm 0.01$ & 0.27 \\
\hline Body fat $(\%)$ & $38.6 \pm 1.5$ & $38.8 \pm 1.4$ & 0.45 & $40.5 \pm 1.2$ & $39.8 \pm 1.2$ & 0.25 \\
\hline Systolic BP (mmHg) & $161 \pm 3$ & $154 \pm 3$ & 0.09 & $163 \pm 5$ & $158 \pm 4$ & 0.31 \\
\hline Diastolic BP (mmHg) & $88 \pm 2$ & $84 \pm 3$ & 0.16 & $93 \pm 2$ & $89 \pm 2$ & 0.17 \\
\hline fP-triacylglycerol (mmol/l) & $0.8 \pm 0.1$ & $0.7 \pm 0.1$ & 0.35 & $0.8 \pm 0.1$ & $0.9 \pm 0.1$ & 0.28 \\
\hline fP-HDL-cholesterol (mmol/l) & $1.9 \pm 0.1$ & $1.8 \pm 0.1$ & 0.70 & $1.9 \pm 0.1$ & $1.8 \pm 0.1$ & 0.66 \\
\hline fP-glucose (mmol/l) & $6.0 \pm 0.2$ & $6.1 \pm 0.2$ & 0.43 & $5.9 \pm 0.2$ & $5.8 \pm 0.2$ & 0.92 \\
\hline fP-insulin (pmol/I) & $57.6 \pm 8.4$ & $57.0 \pm 6.6$ & 0.70 & $56.4 \pm 7.2$ & $58.2 \pm 8.4$ & 0.56 \\
\hline Quadriceps m. mass $(\mathrm{g})^{\mathrm{a}}$ & $139 \pm 4$ & $148 \pm 4$ & 0.01 & $143 \pm 5$ & $162 \pm 6$ & 0.00 \\
\hline Adductor magnus $\mathrm{m}$. mass $(\mathrm{g})^{\mathrm{a}}$ & $92 \pm 4$ & $97 \pm 4$ & 0.06 & $95 \pm 4$ & $97 \pm 4$ & 0.11 \\
\hline Hamstring $\mathrm{m}$. mass $(\mathrm{g})^{\mathrm{a}}$ & $51 \pm 3$ & $50 \pm 3$ & 0.37 & $47 \pm 2$ & $50 \pm 3$ & 0.33 \\
\hline Adductor longus $\mathrm{m}$. mass $(\mathrm{g})^{\mathrm{a}}$ & $27 \pm 2$ & $27 \pm 2$ & 0.59 & $23^{\mathrm{b}} \pm 2$ & $26 \pm 2$ & 0.02 \\
\hline
\end{tabular}

In the OOM group, the sample size for muscle masses at baseline is $n=16$ and after exercise $n=14$

${ }^{\text {a }}$ Muscle compartments refer to depots of 5-6 slices

${ }^{\mathrm{b}}$ Adductor longus muscle mass at baseline is significantly lower in the OOM group than the OLM group $(p=0.03)$

m., muscle 

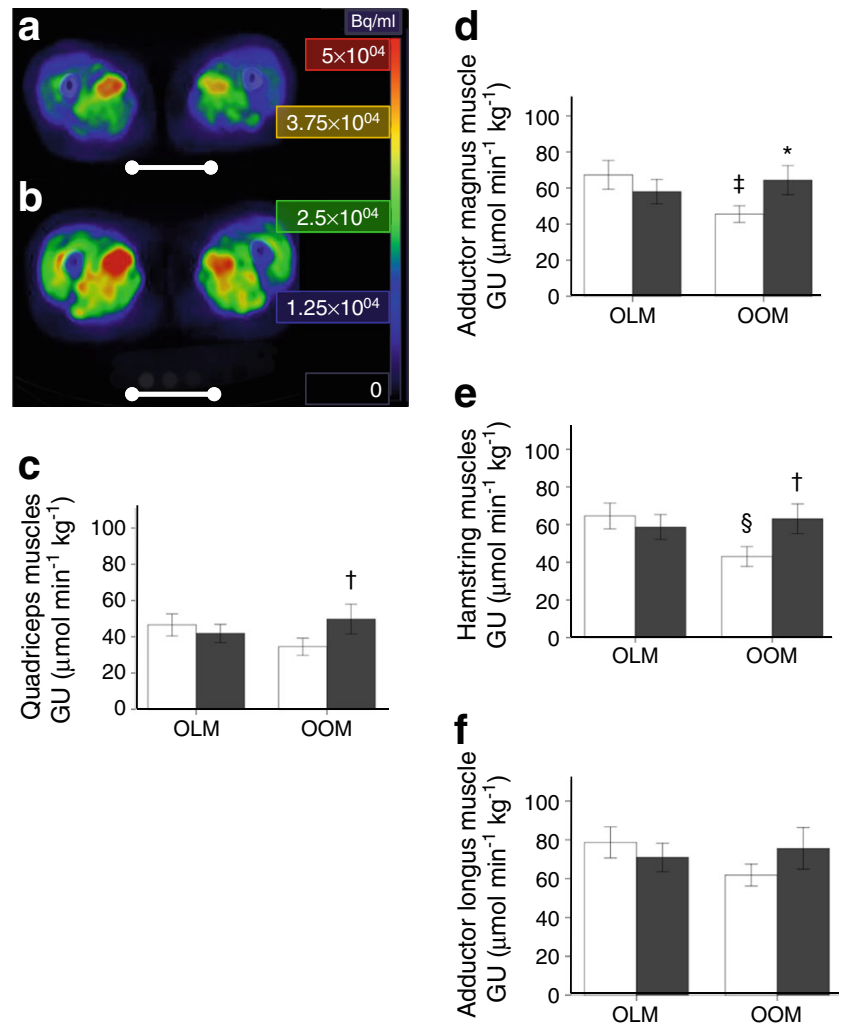

Fig. 5 PET/CT showing ${ }^{18} \mathrm{~F}$-FDG (glucose analogue) uptake in the thighs of an elderly female OOM (a) before and (b) after exercise treatment. Scale bars, $10 \mathrm{~cm}$. Treatment increased sensitivity to the insulin stimulus of the skeletal muscle in the legs. (c-f) Effect of resistance exercise on GU/kg in different muscle compartments, (c) quadriceps muscle, (d) adductor magnus muscle and (e) hamstring muscle were positively affected by the training in the OOM but not in the OLM. Baseline (white bars) vs treatment (black bars): ${ }^{*} p \leq 0.05 ;{ }^{\dagger} p^{\wedge} \leq 0.05$. Between groups: ${ }^{\star} p \leq 0.05 ;{ }^{\circledR} p^{\wedge} \leq 0.05$

0.032; $\rho=-0.61, p^{\wedge}=0.047 ; \rho=-0.66, p^{\wedge}=0.029$, respectively) and negatively with the change in depot GU of quadriceps muscles ( $\rho=-0.75, p^{\wedge}=0.013$; Fig. 7 ) in the OOM group only.

\section{Discussion}

In the present study, we found that high maternal BMI predisposes to skeletal muscle insulin resistance in elderly offspring

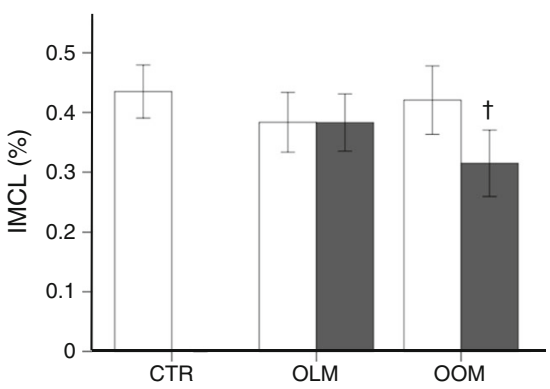

Fig. 6 IMCL decreases after exercise only in the OOM group. Baseline (white bars) vs treatment (black bars): ${ }^{\dagger} p^{\wedge}=0.043$

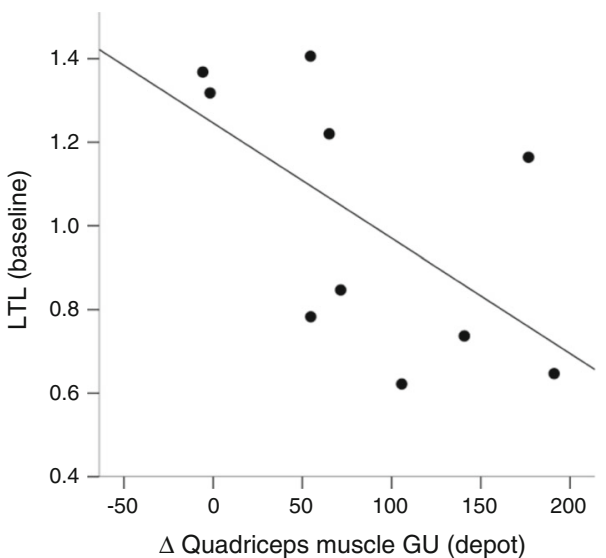

(\%)

Fig. 7 Negative linear relation between LTL at baseline and the change in GU (depot) in the quadriceps muscles in the OOM group. This finding suggests that a shorter telomere length (more unfavourable situation) is associated with a bigger improvement in insulin sensitivity than a longer telomere length

and that the offspring of obese mothers benefit more from RT than the offspring of non-obese mothers. To our knowledge, no study has previously evaluated the effect of maternal BMI on insulin sensitivity in elderly humans.

We studied elderly women with skeletal muscle weakness ('frail') and compared OOM with OLM. Whole body insulin sensitivity was similar between the CTR group and the OLM group (or the whole frail group) and did not differ from previously published values measured in healthy postmenopausal women [31]. Therefore, our aim to find insulin resistance in frail elderly women has not been verified in our study setting; most likely due to the selection criteria used for frailty, as discussed below. However, when insulin sensitivity was measured directly in skeletal muscle, which is the tissue responsible for the majority of insulin-stimulated GU, we found insulin resistance in OOM women. To our knowledge, muscle insulin sensitivity has not been studied directly in elderly people before, but some semi-quantitative studies using imaging have been conducted after walking [32, 33].

The current study also evaluated the effects of RT in elderly women. RT coupled with weight loss improves insulin responses in postmenopausal moderately obese women [19], and RT alone has been shown to be equally effective as aerobic exercise in provoking improvements in glucose metabolism in men [18]. Both RT and aerobic exercise in a mixed group of aged women and men improved whole body insulin sensitivity [34]. We focused on the effects of RT on glucose disposal in the whole body and at the skeletal muscle level in elderly frail women and the interaction with maternal BMI. Marcus et al found that 16 weeks of RT in older adults with type 2 diabetes is beneficial in improving whole body insulin sensitivity and increasing muscle size and FDG uptake (standard uptake value [SUV]) [35]. Cuff et al found that RT in addition to aerobic training enhances glucose disposal in 
postmenopausal women with type 2 diabetes [36]. We found that after RT, whole body and skeletal muscle insulin sensitivity was increased in frail elderly OOM but not in the OLM group. Overall, our data show that frail daughters of obese mothers develop skeletal muscle insulin resistance during ageing and benefit from RT more than daughters of lean mothers. However, the positive associations found between the improvement in insulin sensitivity and the capability to lift weights in the whole frail group support the importance of RT in frail people. OOM might benefit more from the exercise than OLM due to their initially worse metabolic condition.

LTL was positively associated with skeletal muscle insulin sensitivity in OOM, and those with shorter telomere length at baseline showed the biggest improvement in skeletal muscle insulin sensitivity. LTL is inversely associated with type 2 diabetes [14] and insulin resistance in men [15] and premenopausal women but not in postmenopausal women [16]. We used the hyperinsulinaemic-euglycaemic clamp to assess insulin sensitivity, which is the gold standard technique. In line with previous findings, we report a lack of association between insulin resistance and telomere length in the elderly non-frail and frail OLM women. Interestingly, only in OOM, LTL had positive associations with skeletal muscle insulin sensitivity in some of the muscle groups studied.

In a previous study, aerobic exercise did not produce changes in telomere length in postmenopausal women [37]. Our results show that RT did not change telomere length in any of our study groups. However, in the OOM group, shorter telomere length (a more unfavourable situation) was associated with a greater improvement in insulin sensitivity, again supporting the notion that an individual with a worse health status has more room for improvement than an already healthy one.

Interestingly, in elderly women (both frail and non-frail) GU per kg was higher in the adductor longus than in other thigh muscles, especially in OOM. We have not previously found significant differences between insulin-stimulated GU rates in different muscle compartments in young or middleaged individuals $[38,39]$. In line with this finding, similar SUV values in different compartments have been reported recently by Oi et al [40]. Our findings of a highly activated adductor longus muscle at rest might be explained by abnormal postural behaviours, such as a backward disequilibrium [41] in elderly women. This disequilibrium has been hypothesised to be due to a backward tilt in the postural vertical [42]. An elegant study has shown that the adductor longus (together with other leg muscles) is responsible for this reaction to the backward tilt [43]. It could be argued that adductor longus muscle, one of the major muscles involved in balance [44], is particularly solicited to compensate the postural alteration typical of ageing.

IMCL in leg skeletal muscle in the elderly is higher than in younger adults and is correlated to insulin resistance, even after accounting for body fat [45]. In our study, OLM and OOM did not differ for body fat and IMCL, but the exercise treatment decreased IMCL significantly only in the OOM group. The decrement of IMCL following exercise intervention is in line with a previous study in rats [46], but some studies in humans reported increments in IMCL after aerobic exercise $[47,48]$. In a study of elderly men, aerobic but not resistance exercise increased IMCL [49]. The IMCL decrease in the OOM group in our study, together with the improvement in skeletal muscle insulin sensitivity, strength (also in OLM) and mass (also in OLM) testify how elderly 'frail' women benefit from resistance exercise, especially offspring of obese/overweight mothers.

There are limitations in our study. The groups studied were relatively small. However, the study design and techniques used restrict the use of large groups. The groups of frail women had lower handgrip strength but were similar to the CTR group in relation to age, BMI and body fat percentage. Nonetheless, the CTR group had higher quadriceps muscles mass and tended to have better insulin sensitivity than the OOM group. Although the handgrip test is considered a valid marker for frailty $[9,11]$, we cannot exclude that a multi-component definition of frailty could have shown more marked differences. Furthermore, we cannot exclude the influence of genetic background on fetal programming from maternal obesity, but there is solid evidence that fetal programming has a bigger role than genetic background $[5,6]$.

In conclusion, maternal obesity during pregnancy and telomere length are associated with insulin resistance in the elderly offspring, and RT may help to reverse this disadvantage, more so among OOM than in OLM.

Acknowledgements We warmly thank research nurses P. Nyholm (Folkhälsan Research Centre, Helsinki, Finland) and M. Koutu (Turku PET Centre, University of Turku, Turku, Finland) for their work with participant recruitment, logistics and data handling. We thank physiotherapists L. Penttinen and M. Lipasti (Folkhälsan Research Centre) for their valuable contribution during the intervention. We also thank all personnel of the Turku PET Centre.

Funding This study was supported by the EU FP7 grant (no. 278603) for DORIAN project. Additional funding came from the Academy of Finland, the Finnish Cultural Foundation, Central and Varsinais-Suomi Regional fund and the Turku University Foundation. The study was conducted within the Centre of Excellence for Cardiovascular and Metabolic Diseases, supported by the Academy of Finland, University of Turku, Turku University Hospital and Åbo Academy University.

Duality of interest The authors declare that there is no duality of interest associated with this manuscript.

Contribution statement $\mathrm{MB}$ made substantial contributions to conception, design and coordination of the study, acquired the clinical data, prepared leucocytes for analysis, supervised the analysis of PET-images and analysed MRS data, conducted the statistical analysis, interpreted the results and drafted the article. VH made substantial contributions to the study design and acquired the clinical data. MAG analysed and interpreted the data and drafted part of the article. SK acquired clinical 
data, analysed PET-images and drafted part of the article. JRR acquired clinical data and interpreted the results. HL acquired clinical data and revised the manuscript. SA acquired clinical data, prepared leucocytes for analysis, analysed muscle masses and drafted the article. RMB acquired clinical data, prepared leucocytes for analysis and drafted the article. $\mathrm{M}-\mathrm{JH}$ contributed to the statistical analysis and interpretation of the data and drafted the article. JKo acquired clinical data. NS provided substantial contributions to the acquisition of the data and drafted part of the article. MKS made substantial contributions to the conception and design of the study and to participant recruitment. JA analysed and interpreted the data and drafted the article. JKu analysed and interpreted the data and drafted the article. SS made substantial contributions to the conception and design of the study, to participant recruitment and to interpretation of the data, and drafted the article. PI, JGE and PN made substantial contributions to the conception, design and coordination of the study and to interpretation of the data, and drafted the article. All authors revised the article critically for important intellectual content and approved the final version to be published. MB is the guarantor of this work and, as such, had full access to all the data in the study and takes responsibility for the integrity of the data and the accuracy of the data analysis.

\section{References}

1. Catalano PM, Presley L, Minium J, Hauguel-de Mouzon S (2009) Fetuses of obese mothers develop insulin resistance in utero. Diabetes Care 32:1076-1080

2. Eriksson JG, Sandboge S, Salonen MK, Kajantie E, Osmond C (2014) Long-term consequences of maternal overweight in pregnancy on offspring later health: findings from the Helsinki Birth Cohort Study. Ann Med 46:434-438

3. Mingrone G, Manco M, Mora ME et al (2008) Influence of maternal obesity on insulin sensitivity and secretion in offspring. Diabetes Care 31:1872-1876

4. Gilbert JS, Nijland MJ (2008) Sex differences in the developmental origins of hypertension and cardiorenal disease. Am J Physiol Regul Integr Comp Physiol 295:R1941-R1952

5. Obregon MJ (2010) Maternal obesity results in offspring prone to metabolic syndrome. Endocrinology 151:3475-3476

6. Vaag A, Brons C, Gillberg L et al (2014) Genetic, nongenetic and epigenetic risk determinants in developmental programming of type 2 diabetes. Acta Obstet Gynecol Scand 93:1099-1108

7. Gluckman PD, Hanson MA, Cooper C, Thornburg KL (2008) Effect of in utero and early-life conditions on adult health and disease. N Engl J Med 359:61-73

8. Danielzik S, Langnase K, Mast M, Spethmann C, Muller MJ (2002) Impact of parental BMI on the manifestation of overweight 5-7 year old children. Eur J Nutr 41:132-138

9. Abbatecola AM, Paolisso G (2008) Is there a relationship between insulin resistance and frailty syndrome? Curr Pharm Des 14:405410

10. Fried LP, Tangen CM, Walston J et al (2001) Frailty in older adults: evidence for a phenotype. J Gerontol Ser A Biol Med Sci 56: M146-M156

11. Syddall H, Cooper C, Martin F, Briggs R, Aihie Sayer A (2003) Is grip strength a useful single marker of frailty? Age Ageing 32:650 656

12. Rowe JW, Minaker KL, Pallotta JA, Flier JS (1983) Characterization of the insulin resistance of aging. J Clin Invest 71:1581-1587

13. von Zglinicki T, Martin-Ruiz CM (2005) Telomeres as biomarkers for ageing and age-related diseases. Curr Mol Med 5:197-203

14. Hovatta I, de Mello VD, Kananen L et al (2012) Leukocyte telomere length in the Finnish Diabetes Prevention Study. PLoS One 7: e34948
15. Al-Attas OS, Al-Daghri NM, Alokail MS et al (2010) Adiposity and insulin resistance correlate with telomere length in middle-aged Arabs: the influence of circulating adiponectin. Eur J Endocrinol 163:601-607

16. Aviv A, Valdes A, Gardner JP, Swaminathan R, Kimura M, Spector TD (2006) Menopause modifies the association of leukocyte telomere length with insulin resistance and inflammation. J Clin Endocrinol Metab 91:635-640

17. DeFronzo RA, Tobin JD, Andres R (1979) Glucose clamp technique: a method for quantifying insulin secretion and resistance. Am J Physiol 237:E214-E223

18. Ferrara CM, Goldberg AP, Ortmeyer HK, Ryan AS (2006) Effects of aerobic and resistive exercise training on glucose disposal and skeletal muscle metabolism in older men. J Gerontol Ser A Biol Med Sci 61:480-487

19. Ryan AS, Pratley RE, Goldberg AP, Elahi D (1996) Resistive training increases insulin action in postmenopausal women. J Gerontol Ser A Biol Med Sci 51:M199-M205

20. Iozzo P, Holmes M, Schmidt MV et al (2014) Developmental ORIgins of Healthy and Unhealthy AgeiNg: the role of maternal obesity - introduction to DORIAN. Obes Facts 7:130-151

21. Yliharsila H, Kajantie E, Osmond C, Forsen T, Barker DJ, Eriksson JG (2007) Birth size, adult body composition and muscle strength in later life. Int J Obes 31:1392-1399

22. Provencher SW (1993) Estimation of metabolite concentrations from localized in vivo proton NMR spectra. Magn Reson Med 30:672-679

23. Bucci M, Borra R, Nagren K et al (2011) Human obesity is characterized by defective fat storage and enhanced muscle fatty acid oxidation, and trimetazidine gradually counteracts these abnormalities. Am J Physiol Endocrinol Metab 301: E105-E112

24. Hamacher K, Coenen HH, Stocklin G (1986) Efficient stereospecific synthesis of no-carrier-added 2-[18F]-fluoro-2-deoxy-D-glucose using aminopolyether supported nucleophilic substitution. J Nucl Med 27:235-238

25. Gjedde A (1982) Calculation of cerebral glucose phosphorylation from brain uptake of glucose analogs in vivo: a re-examination. Brain Res 257:237-274

26. Patlak CS, Blasberg RG, Fenstermacher JD (1983) Graphical evaluation of blood-to-brain transfer constants from multiple-time uptake data. J Cereb Blood Flow Metab 3:1-7

27. Peltoniemi P, Lonnroth P, Laine $\mathrm{H}$ et al (2000) Lumped constant for [(18)F]fluorodeoxyglucose in skeletal muscles of obese and nonobese humans. Am J Physiol Endocrinol Metab 279:E1122E1130

28. Kanof ME, Smith PD, Zola H (1996) Isolation of whole mononuclear cells from peripheral blood and cord blood. Curr Protoc Immunol Chapter 7: Unit 7.1

29. Cawthon RM (2009) Telomere length measurement by a novel monochrome multiplex quantitative PCR method. Nucleic Acids Res 37: e21

30. Benjamini Y, Hochberg Y (1995) Controlling the false discovery rate: a practical and powerful approach to multiple testing. J R Stat Soc Ser B Methodol 57:289-300

31. Ferrara CM, Goldberg AP, Nicklas BJ, Sorkin JD, Ryan AS (2008) Sex differences in insulin action and body fat distribution in overweight and obese middle-aged and older men and women. Physiol Appl Nutr Metab 33:784-790

32. Shimada H (2012) Imaging of glucose uptake during walking in elderly adults. Curr Aging Sci 5:51-57

33. Shimada H, Kimura Y, Lord SR et al (2009) Comparison of regional lower limb glucose metabolism in older adults during walking. Scand J Med Sci Sports 19:389-397 
34. Consitt LA, Van Meter J, Newton CA et al (2013) Impairments in site-specific AS160 phosphorylation and effects of exercise training. Diabetes 62:3437-3447

35. Marcus RL, Addison O, LaStayo PC et al (2013) Regional muscle glucose uptake remains elevated one week after cessation of resistance training independent of altered insulin sensitivity response in older adults with type 2 diabetes. J Endocrinol Investig 36:111-117

36. Cuff DJ, Meneilly GS, Martin A, Ignaszewski A, Tildesley HD, Frohlich JJ (2003) Effective exercise modality to reduce insulin resistance in women with type 2 diabetes. Diabetes Care 26: 2977-2982

37. Mason C, Risques RA, Xiao L et al (2013) Independent and combined effects of dietary weight loss and exercise on leukocyte telomere length in postmenopausal women. Obesity 21:E549-E554

38. Nuutila P, Knuuti MJ, Heinonen OJ et al (1994) Different alterations in the insulin-stimulated glucose uptake in the athlete's heart and skeletal muscle. J Clin Invest 93:2267-2274

39. Nuutila P, Maki M, Laine $\mathrm{H}$ et al (1995) Insulin action on heart and skeletal muscle glucose uptake in essential hypertension. J Clin Invest 96:1003-1009

40. Oi N, Iwaya T, Itoh M, Yamaguchi K, Tobimatsu Y, Fujimoto T (2003) FDG-PET imaging of lower extremity muscular activity during level walking. J Orthop Sci 8:55-61

41. Manckoundia P, Mourey F, Perennou D, Pfitzenmeyer P (2008) Backward disequilibrium in elderly subjects. Clin Interv Aging 3: $667-672$

42. Manckoundia P, Mourey F, Pfitzenmeyer P, Van Hoecke J, Perennou D (2007) Is backward disequilibrium in the elderly caused by an abnormal perception of verticality? A pilot study. Clin Neurophysiol 118:786-793

43. Henry SM, Fung J, Horak FB (1998) EMG responses to maintain stance during multidirectional surface translations. J Neurophysiol 80:1939-1950

44. Green DL, Morris JM (1970) Role of adductor longus and adductor magnus in postural movements and in ambulation. Am J Phys Med 49:223-240

45. Cree MG, Newcomer BR, Katsanos CS et al (2004) Intramuscular and liver triglycerides are increased in the elderly. J Clin Endocrinol Metab 89:3864-3871

46. Vaisy M, Szlufcik K, De Bock K et al (2011) Exercise-induced, but not creatine-induced, decrease in intramyocellular lipid content improves insulin sensitivity in rats. J Nutr Biochem 22:1178-1185

47. Dube JJ, Amati F, Stefanovic-Racic M, Toledo FG, Sauers SE, Goodpaster BH (2008) Exercise-induced alterations in intramyocellular lipids and insulin resistance: the athlete's paradox revisited. Am J Physiol Endocrinol Metab 294:E882-E888

48. Haus JM, Solomon TP, Lu L et al (2011) Intramyocellular lipid content and insulin sensitivity are increased following a shortterm low-glycemic index diet and exercise intervention. Am J Physiol Endocrinol Metab 301:E511-E516

49. Ngo KT, Denis C, Saafi MA, Feasson L, Verney J (2012) Endurance but not resistance training increases intra-myocellular lipid content and beta-hydroxyacyl coenzyme A dehydrogenase activity in active elderly men. Acta Physiol 205:133-144 